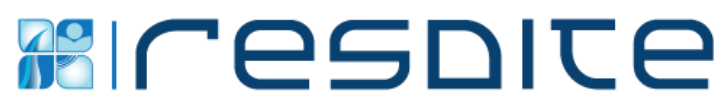

\section{R.A.I.O - espaço virtual para a construção colaborativa do cuidado em saúde no processo de adoecer por câncer}

\author{
R.A.I.O - virtual space for the collaborative construction of health care in the process of \\ becoming ill for cancer
}

\section{Yuri Hamirani Gonçalves da Silva1, Heloísa Cristina Figueiredo Frizzo²}

\begin{abstract}
Resumo
Introdução e objetivo: Este estudo objetiva compreender o processo de construção colaborativa de um espaço virtual para a expressão de emoções e sentimentos, produção de cuidado integrado, interação social e criação de uma rede de apoio/suporte à pessoa com diagnóstico de câncer. Na atualidade, tem-se observado que as redes sociais e a internet são utilizadas como ferramentas para o suporte e melhoria da qualidade de vida das pessoas em situação de adoecimento, em um movimento de ressignificação deste processo. Método: Trata-se de uma pesquisa qualitativa, transversal, a partir da pesquisa-ação, realizada junto aos profissionais e usuários de um serviço especializado em Oncologia, por meio da utilização da internet. Resultados: A criação e produção colaborativa de um site temático sobre o câncer facilitou o acesso à informação qualificada, assim como a revisão da relação terapêutica entre profissionais e usuários da unidade de oncologia, cenário da pesquisa. Também contribuiu para a expressão da relevância do tema religiosidade/espiritualidade no processo de adoecer por câncer. Conclusão: Acredita-se que promover espaços de socialização e produção de vida, ainda que no mundo virtual, tende a contribuir para agregar benefícios para a melhoria da sistematização da atenção em saúde. Além disso, promove-se autocuidado, empoderamento e autogestão em saúde.
\end{abstract}

Palavras-Chave: E-saúde. Câncer. Mídias Sociais.

\section{Abstract}

Introduction and objective: This study aims to understand the process of collaborative construction of a virtual space to express emotions and feelings, production of integrated care, social interaction and creation of support network for the person diagnosed with cancer. Nowadays, has been observed that social networks and the internet have been used as tools to support and improve the quality of life of people suffering from illness, in a process of resignification of this process. Method: This is a qualitative, cross-sectional research based on the action research carried out with professionals and users of a specialized Oncology service through the use of the internet. Results: The creation and collaborative production of a cancer site facilitated the access to qualified information, as well as for the review of the professionals therapeutic relationship and users of the oncology unit, the research scenario. It also contributed to the expression of the relevance of the theme religiosity/spirituality in the process of becoming cancer. Conclusion: It is believed that promoting spaces of socialization and production of life, although in the virtual world, try to contribute to add benefits for the

1 Graduando em Terapia Ocupacional. Universidade Federal do Triângulo Mineiro (UFTM). Email: yuri_hamirani@hotmail.com 2 Doutora. Departamento de Terapia Ocupacional da Universidade Federal do Triângulo Mineiro (UFTM). Email:

heloisa.frizzo@yahoo.com.br

Correspondência: Departamento de Terapia Ocupacional UFMT - Centro de Pesquisas Prof. Aluízio Rosa Prata - Rua Vigário Carlos, 100 - 4andar, sala 438. Bairro Abadia - Uberaba - MG, Brasil. CEP: 38025-350. 
improvement of the systematization of health care. In addition, it promotes self-care, empowerment and self-management in health.

Keywords: E- health. Cancer. Social Media.

\section{Introdução}

A rede social é uma construção social que permite que indivíduos, empresas e entidades estejam conectados ou interligados e que estabeleçam relações de afetos e de trocas diversas, por meio de conexões de amizades, empatia e comerciais. Assim, as redes sociais destacam-se por um fluxo social que une indivíduos com interesses em comum ${ }^{1 ; 2}$.

Dentre os autores que estudam estes fenômenos, destaca-se Manuel Castells $^{3}$, que contribui diretamente com a discussão sobre sociedade em rede e aponta que vivemos em uma era de informação e interação, e por este motivo, nos chama atenção para o fluxo social destas informações e seu impacto na sociedade, cultura e modo de produção.

Ademais, para esse autor, os usuários da internet ingressam em redes ou grupos online e isso ocorre por meio de interesses e valores em comum que são intrínsecos para cada indivíduo 3 .

Desse modo, tanto offline, no mundo real, quanto online, nas redes sociais, a internet tende a facilitar a ligação de pessoas com diversas características sociais ${ }^{3}$. A utilização desse recurso virtual possibilita ao indivíduo lidar com situações adversas, à medida em que pode acionar elementos do seu entorno social para poder solucioná-las ${ }^{4 ; 5}$.

Dessa forma, as redes sociais podem proporcionar apoio social aos indivíduos no que se refere à ajuda mútua que pode ser potencializada quando estas são fortes e integradas. Salienta-se que o apoio social fornecido pelas redes demonstra aspectos positivos das relações sociais, como o compartilhar informações, o auxílio em momentos de crise e o aumento da presença em movimentos sociais, resultados estes criados e estabelecidos por conexões entre indivíduos ${ }^{4}$.

Além disso, as redes sociais não abarcam apenas movimentos sociais, mas também organizações não governamentais (ONGs), ambientes acadêmicos, governos e organizações internacionais. Em todas essas situações as fronteiras das redes são definidas com base em ações e valores comuns que reúnem indivíduos de diferentes instituições. Assim, apesar de incluírem atores normalmente não identificados como parte de movimentos sociais, essas redes possuem três características relevantes: interações informais, ações em 
comum em torno de conflitos políticos ou culturais, e identidade coletiva ${ }^{6}$.

Compreendendo-se as características acima destacadas em relação ao conceito de rede social, esta pesquisa teve por objetivo a criação de uma mídia digital temática, em formato de site, especializada na vivência do cotidiano de pessoas diagnosticadas e em tratamento de câncer. Acredita-se que esta mídia social possa, ao longo de sua utilização, consolidar-se como uma rede de interação social entre pessoas com diagnóstico de câncer, seus familiares e cuidadores, com tendência a evoluir para uma rede de apoio e suporte para estas pessoas em adoecimento e tratamento, constituindo-se como uma estratégia de enfrentamento no processo de saúdedoença e em suas repercussões.

A literatura aponta que 0 enfrentamento do diagnóstico de câncer faz demandas não somente ao paciente, mas também da família e de seus cuidadores, em função dessa enfermidade ser um advento complexo e multidimensional. Neste contexto, a participação e envolvimento dos familiares e pessoas próximas à pessoa com câncer tende a ser relevante, uma vez que propicia o fortalecimento de vínculos e estes repercutem na vida e no cotidiano do paciente acometido por essa doença?

A complexidade e a extensão das repercussões clínicas do câncer na vida do indivíduo tem levado muitos autores a desenvolverem estudos com o propósito de analisar o impacto e consequências dessa condição. Nesse caso, verifica-se que todas as ações de ajuda às pessoas que apresentam condições crônicas de saúde envolvem o enfrentamento do problema de maneira individual, e se faz necessária a adaptação psicossocial que pode ser realizada por meio do desenvolvimento de ações que possam auxiliar esses indivíduos a lidarem melhor com essa situação de saúde em que se encontram ${ }^{8}$.

Considerando-se ainda a complexidade, a multidimensionalidade do processo de adoecer por câncer, o tratamento e as repercussões da doença na vida e no cotidiano da pessoa, justificase a realização de estudos que priorizem a escuta das necessidades e vivências por eles experimentadas na busca do reestabelecimento da integridade biológica, psicológica e social. Esta compreensão tende a contribuir para a melhoria da assistência a esta população ${ }^{9}$.

No esforço de ampliar a discussão no que se refere à atenção biopsicossocial à pessoa com diagnóstico de câncer, esta pesquisa objetivou criar um espaço virtual para a expressão da vivência do adoecer por câncer e suas repercussões. Assim, a questão norteadora desse estudo foi: "Como a construção colaborativa de uma mídia social temática pode se estabelecer como um espaço para a interação social e criação de redes de apoio/suporte mediante o adoecer por câncer?" 


\section{Método}

Trata-se de uma pesquisa qualitativa, transversal $^{10}$, a partir da pesquisa-ação, que é definida por Michel Thiollent ${ }^{11}$ como um tipo de pesquisa com base empírica que é concebida e realizada em estreita associação com uma ação ou com a resolução de um problema coletivo, no qual os pesquisadores e os participantes representativos da situação ou do problema estão envolvidos de modo cooperativo ou participativo.

Este estudo foi realizado em uma Unidade de Oncologia, vinculada a um Hospital de Clínicas do Interior do Estado de Minas Gerais.

Esta Unidade de Oncologia está estruturada para cuidados ambulatoriais de pacientes com câncer com abrangência regional. Suas atividades de assistência estão organizadas por consultas realizadas por médicos, enfermagem oncológica, assistência social, psicologia, nutrição e terapia ocupacional.

A área de abrangência desse local é a administração de terapêuticas e cuidados de suporte com a finalidade de oferecer um cuidado humanizado e integral aos pacientes com câncer, oferecendo procedimentos médicos e de enfermagem que não requeiram internação, como administração de medicamentos, coleta de amostras biológicas e execução de exames médicos específicos. O local inclui sala de espera (1); recepção (1); consultórios (9); brinquedoteca (1); postos de enfermagem (2); secretaria (1); área administrativa (1); cozinha (1); e enfermarias (5).

Esta pesquisa foi desenvolvida de março de 2018 a março de 2019, em duas etapas. Na primeira etapa, foi construído um ambiente virtual, em formato de site, produzido com base na literatura sobre tecnologias de comunicação, informação e oncologia que permitiu a criação da estrutura do site com diferentes links temáticos afins. Na segunda, este produto virtual permitiu o planejamento e a produção de dados colaborativos disponibilizados no próprio site, que posteriormente foram utilizados para a categorização e análise dos dados.

Os resultados desta pesquisa foram sistematizados em categorias de análise segundo os links elencados para o site, a saber: "Home", "Álbum de histórias", "Cuidando de mim", "Buscando informações", "Depoimentos" e "Contato".

Para fins de produção dos conteúdos e postagens, houve a inserção do pesquisador no campo/cenário do estudo, por se tratar de uma pesquisa participante, que exige a inserção do pesquisador no ambiente natural de ocorrência do fenômeno e de sua interação com a situação investigada ${ }^{10}$, promovendo estratégias para a sensibilização dos profissionais. Essa sensibilização estimulou o movimento de participação e a produção de conteúdos temáticos afins, buscando o envolvimento dos profissionais na construção do espaço colaborativo. 
Ressalta-se que os profissionais, uma vez sensibilizados a colaborar com o espaço virtual, ficaram livres para abordar temas de sua escolha, devendo levar em consideração a temática do próprio sítio eletrônico, o adoecer por câncer e suas repercussões.

Esta pesquisa está pautada nos preceitos éticos da resolução 510/2016, de acordo com as normativas de uso de informações de acesso público, aberto, sem identificação pessoal.

\section{Resultados}

Os resultados a seguir descritos são originários das etapas: construção da mídia digital em formato sítio eletrônico (site); produção colaborativa de conteúdo para a mídia digital.

\section{A construção da mídia digital em formato de sítio eletrônico (site)}

Esta etapa foi realizada tendo como norte a literatura afim sobre tecnologias de comunicação, informação e oncologia.

Para fins da construção do site foi utilizada a plataforma www.wixsite.com, que hospeda sítios eletrônicos gratuitamente e fornece suporte para adquirir domínios pagos.

Dentre os referenciais teóricos que subsidiaram a produção da mídia digital sobre o adoecer em virtude do câncer e suas repercussões, destacam-se: Promoção de saúde, Expressão de emoções e sentimentos, Rotina e cotidiano, Tratamentos, Dúvidas e a Produção de vida.

Assim, foi possível a construção do sítio eletrônico intitulado "R.A.I.O" (Rede de Apoio ao Indivíduo na Oncologia), disponível no endereço eletrônico "www.raiooncologia.wixsite.com/raio", constituído por seis (06) sessões que estão descritas e caracterizadas a seguir:

A sessão "Home" refere-se à página de rosto do site. Trata-se da página de apresentação e entrada. Nesta página de entrada são disponibilizadas atualizações e novidades, esclarecimentos sobre o objetivo e motivação para a criação do site, além da divulgação do trabalho realizado e descrição de seus criadores, tutores e autores.

$\mathrm{Na}$ sessão "Álbum de histórias" (Anexo 1), são disponibilizados depoimentos de profissionais de saúde e usuários de serviços de oncologia em relação a vivência do adoecer e cuidar de pessoas com diagnóstico de câncer, além de indicações de leituras, histórias de experiências pessoais e produções de vida.

Para os pesquisadores, esta sessão teve como propósito inicial o apoio social e a instilação de esperança nos pacientes com câncer por meio da integração grupal que passou a ocorrer nesse espaço virtual que se configurou como modificador positivo na qualidade de vida e no apoio ao indivíduo com câncer. Esse dado vai ao encontro do estudo de Cohen ${ }^{12}$, segundo o qual o apoio social favorece a regulação 
emocional, alivia experiências emocionais estressantes, bem como passa a ser uma fonte de apoio informacional aos pacientes acometidos por essa enfermidade. Ademais, este estudo enfatiza que informações sobre a doença são um aspecto central da prestação de cuidados de qualidade ${ }^{12}$.

A sessão "Cuidando de mim" (Anexo 2) é um espaço virtual dedicado às ações desenvolvidas por profissionais de saúde e usuários para a produção de vida e projetos relacionados ao processo de adoecer por câncer.

Nesse espaço, o autocuidado é explorado como uma elaboração essencial para vida do paciente com câncer, tendo como apoio a produção do que lhe faz sentir vivo. Cabe ressaltar que 0 autocuidado pode ser definido como uma atividade do indivíduo apreendida pelo mesmo e orientada para um fim. É uma ação realizada ou desenvolvida em uma situação concreta de vida em que o indivíduo se dirige para si mesmo, servindo também para regular fatores que afetam diretamente 0 seu próprio desenvolvimento pessoal e podem ser compreendidos como atividades em benefício da sua vida, de sua saúde e bemestar ${ }^{13}$. A finalidade desta seção é possibilitar postagens para encorajamento e compartilhamento de ações dos pacientes com câncer quanto a suas realizações pessoais e de autocuidado.

A sessão "Buscando informações" (Anexo 3), tem por objetivo esclarecer dúvidas sobre o câncer e buscar ampliar o acesso à informação em saúde. Para produção do material a ser disponibilizado nesta sessão são necessários o levantamento prévio de evidências científicas acerca de um tema de interesse comum identificado na rotina ou um questionamento realizado por um usuário do serviço via e-mail, para posterior a sistematização da assistência em oncologia. Todo processo de produção desse material é fruto de pesquisa científica criteriosa sobre determinado tema de interesse individual e/ou coletivo relacionado ao adoecer com câncer em bases de dados confiáveis e respeitadas no âmbito científico.

Esta sessão se ampara no termo “Telessaúde", pertencendo a uma contínua busca pela melhoria da qualidade de vida e acesso à informação de qualidade. Pode ser compreendida como uma cadeia produtiva de saúde e um processo natural em que as novas tecnologias são usadas para promover ações que se integram com a qualidade de vida de uma população, atingindo assim a literacia em saúde e a informação nessa área. Essa prática tem tido grande repercussão na prevenção de doenças e promoção de saúde. Isso possibilita a criação de uma rede de aprendizagem colaborativa, na qual se faz uso de tecnologias interativas para ampliar as possibilidades de construção de conhecimento ${ }^{14}$.

A sessão "Depoimentos" (Anexo 4), foi criada para contemplar a oportunidade 
de compartilhamento de vivências pessoais relacionadas ao processo de adoecimento e tratamento decorrentes do câncer. Acredita-se que a possibilidade de compartilhar experiências pode favorecer a criação de laços e apoio/suporte por meio da instilação de esperança ao contemplar depoimentos que expressem os desafios do processo de adoecimento e tratamento, incluindo medos, expectativas, superação, fracassos, dentre outras experiências vivenciadas no enfrentamento desta situação.

A sessão "Contato" (Anexo 5), tem por finalidade inicial constituir-se como um canal de comunicação entre os tutores do sítio eletrônico e pessoas com interesses comuns relacionados ao tema Oncologia. Também permite a aproximação e comunicação entre serviço de assistência prestada pela Unidade de Oncologia/Universidade Federal do Triângulo Mineiro (UFTM), em especial com os profissionais de saúde. Ao criar um meio para a interação entre usuários e serviço de assistência em saúde acreditase que é possível fortalecer o desenvolvimento de vínculos e favorecer uma relação de confiança.

As sessões: "Depoimento" e "Contato", promovem a interação com o usuário em potencial, por meio do ambiente virtual, como forma de estreitar relações e contatos, sendo um canal de comunicação.

Para considerar a comunicação mediada pela tecnologia e consumada por intermédio do computador, via internet, podemos nos embasar segundo as perspectivas de Lévy ${ }^{15}$, de acordo com as quais o computador se tornou um dispositivo técnico através do qual a pessoa percebe o mundo, e isto não ocorre somente em um plano empírico, mas também em um plano transcendental, pois cada vez mais o ser humano concebe o social, os seres vivos ou os processos cognitivos por meio de uma matriz de leitura informatizada. Percebe-se, assim, que a comunicação é facilitada por meio da tecnologia.

Por fim, o site intitulado "R.A.I.O" foi planejado e elaborado nos meses de maio a julho de 2018, sendo este último mês em que foi efetivamente disponibilizado, com suas diferentes sessões e fins específicos, acreditando-se que pode contribuir para o empoderamento de pessoas, familiares, cuidadores e profissionais de saúde frente aos desafios vivenciados no processo de enfrentamento de um câncer.

A abordagem do empoderamento do indivíduo discute sobre o conjunto de medidas, técnicas e procedimentos que envolvem adoecimento e ética profissional. Assim, essa abordagem pode ser compreendida como uma proposta para melhorar o relacionamento entre os indivíduos com câncer e os serviços de assistência em saúde. É com essa estratégia básica que se visa a melhoria nos resultados do tratamento $\mathrm{e}$ enfrentamento dessa enfermidade ${ }^{16}$. 


\section{A produção colaborativa de conteúdo para a mídia digital}

Esta etapa consiste na sensibilização dos diferentes profissionais e atores em potencial para a produção do material a ser disponibilizado no sítio eletrônico. Inicialmente houve uma busca ativa para despertar o interesse em compor e produzir o conteúdo para o site, a fim de buscar o envolvimento dos participantes de modo colaborativo.

A produção colaborativa de conteúdo para a mídia digital ocorreu a partir do mês de junho de 2018, por meio da inserção e participação dos pesquisadores no cenário do estudo, neste caso, a Unidade de Oncologia/UFTM. Esta inserção permitiu o estímulo à participação de profissionais e usuários do serviço para a produção colaborativa de temas e conteúdos afins para postagem no site. Até o presente momento (abril/2019) foram postadas cinco produções nas sessões "Álbum de histórias" e "Cuidando de mim", relacionadas aos seguintes temas: "Trajetória e produção de vida", "Nutrição em oncologia", "Câncer" e "Espiritualidade".

A seguir serão descritos resumidamente os temas e assuntos abordados, seguidos de uma análise de conteúdo.

O tema "Trajetória e produção de vida", refere-se ao depoimento, em formato de vídeo e textual, de duas profissionais de saúde que compõem a equipe no serviço de oncologia. Esta ferramenta favoreceu o estreitamento dos laços entre profissionais e os possíveis usuários do sítio eletrônico e da unidade, sendo disponibilizada na sessão "Álbum de histórias". Os depoimentos referem-se às trajetórias de vida de duas profissionais das áreas de enfermagem e medicina, onde destacam suas experiências de vida, de formação pessoal e acadêmica, e de atuação na área da oncologia.

Na postagem, observa-se o relato de um profissional da área médica, ao elucidar uma experiência pessoal de contato com o livro "O pequeno médico", escrito por Graziela Gillioli17 ${ }^{17}$ que destaca o impacto do conteúdo dessa obra literária no manejo das intervenções clínicas.

Este livro aborda a experiência de uma mãe na trajetória de adoecimento por câncer de seu filho, desde o diagnóstico até a morte e as repercussões da perda e do luto. $\mathrm{Na}$ postagem, o profissional aborda a necessidade de humanização da assistência, a partir de autorreflexão sobre a prática profissional, com ênfase na mudança de foco na assistência centrada na pessoa e não na doença. Esta reflexão, por sua vez, possibilitou o diálogo do profissional consigo mesmo, por meio da revisão de suas ações no relacionamento com seus pacientes. Corroborando o estudo de Morais et al. ${ }^{18}$, que aponta que os profissionais de saúde, em especial os que trabalham na área da Oncologia, constantemente experimentam situações difíceis ao mesmo tempo em que são portadores de más notícias. Tal situação, 
na maioria das vezes, leva o trabalhador ao estresse e ao sentimento de solidão, que pode ser minimizado com diálogos que são importantes instrumentos de promoção da saúde. Destaca-se que esses diálogos colocam o profissional diante de si, permitindo seu autoconhecimento, 0 que possibilita melhorias em seu relacionamento com pacientes. Ademais, os profissionais que trabalham com pacientes com câncer necessitam de suporte psicológico para poderem cuidar de si mesmos e expressar seus sentimentos diante das dificuldades que encontram no exercício da sua atividade profissional, no intuito de ofertar um cuidado integral e humanizado a esses indivíduos enfermos, bem como aos seus familiares.

Nota-se também que o livro despertou o profissional para uma valorização do trabalho multi e interprofissional.

Observa-se que esta mudança na perspectiva da relação entre profissional e paciente tende a promover transformações nas relações de poder entre os usuários e os profissionais de um serviço de saúde, com vistas à valorização e autonomia dos mesmos, na construção de uma relação de confiança, pois a confiança faz com que a comunicação entre paciente e profissionais de saúde seja facilitada, levando a um melhor envolvimento do paciente com seu tratamento ${ }^{18}$.

Desta forma, a publicação teve por finalidade sensibilizar profissionais e pacientes de modo a gerar uma melhora em suas relações, contribuindo de forma geral a instilar no paciente a esperança de cura ou de que as coisas possam ser diferentes, a partir da convivência com pessoas que vivem situações semelhantes ${ }^{19}$.

Os temas "Nutrição em oncologia e o "Câncer" foram disponibilizados na sessão "Cuidando de mim", tendo como objetivos orientar e informar acerca de cuidados em saúde. Para a produção do tema "Nutrição em oncologia", inicialmente foi realizada uma entrevista com uma profissional da área de nutrição convidada a falar sobre esta temática de forma acessível e de fácil compreensão.

Esta entrevista foi editada e disponibilizada em formato de vídeo e textual, contextualizando o tema para a oncologia. Assim, acredita-se que esta postagem pôde contribuir para a desmistificação de correlações equivocadas de senso popular em relação à nutrição e câncer. Essa informação em saúde tem por finalidade proporcionar melhoria da qualidade de vida aos pacientes com câncer por meio do acesso à informação qualificada e validada no intuito de melhorar 0 desempenho nutricional desses indivíduos e consequentemente seu autocuidado em saúde.

Salienta-se que a avaliação da qualidade de vida perpassa fatores fisiológicos, emocionais e econômicos. Desse modo, a qualidade de vida auxilia de 
maneira eficaz nos efeitos negativos causados pelas repercussões clínicas do tratamento do câncer, por isto a qualidade de vida e a instrução qualificada é responsável pela melhoria do prognóstico de diversos tipos de câncer, culminando no aumento da sobrevida de pacientes oncológicos $^{20}$. Neste sentido, o Ministério da Saúde ${ }^{21}$ enfatiza que a preservação e/ou melhora do estado nutricional tem um efeito importante na qualidade de vida e no bem-estar dos pacientes com câncer.

O tema "Espiritualidade" foi disponibilizado na sessão "Álbum de histórias", em duas publicações, uma sendo de caráter científico, com base na dissertação de mestrado ${ }^{22}$ de uma das profissionais, e a outra pela indicação de um livro espírita que favoreceu a mudança de perspectiva de um dos profissionais que também faz parte da equipe de saúde na unidade de oncologia pesquisada.

Quando se aborda o tema espiritualidade é essencial diferenciar os termos espiritualidade e religiosidade. A espiritualidade remete à busca de um significado ou sentido da vida, não necessariamente vinculados à crença em um ser supremo. A religiosidade, por sua vez, se encontra no âmbito subjetivo da religião, implicando na relação do indivíduo com um ser transcendente ou superior ${ }^{23}$; 24.

Nota-se que tal tema é pertinente ao sítio eletrônico desenvolvido, pois, segundo a Organização Mundial de Saúde, a espiritualidade é um fator que integra o bem-estar biopsicossocial de um indivíduo, tendo de ser levada em conta para a qualidade de vida e saúde de um indivíduo e/ ou população ${ }^{25}$.

Para Puchalski²6, o conceito de espiritualidade é encontrado em todas as culturas e sociedades. Ela se expressa na busca individual de um sentido por meio da religião e crença em Deus, família, naturalismo, racionalismo, humanismo e, até mesmo, as artes. Todos esses fatores podem influenciar na forma como os indivíduos envolvidos com o seu processo de adoecer percebem sua saúde e sua própria doença e como eles interagem e se posicionam uns com os outros a respeito disso. Assim, nota-se a importância da religiosidade no enfrentamento de doenças como o câncer.

Cabe destacar que a religiosidade/espiritualidade tende a ser reconhecida como recurso para a redução das incertezas decorrentes do adoecimento por câncer. Frente a este diagnóstico é comum a pessoa acometida por essa enfermidade realizar reflexões sobre o sentido da vida e revisar seu sistema de crenças e valores relacionados a esses aspectos religiosos e espirituais.

Observa-se que até o presente momento não houve produções exclusivas de usuários do serviço de saúde e de pessoas usuárias do site. Este fato tende a se constituir como uma limitação do estudo. No entanto, os pesquisadores, conscientes da relevância e do potencial do espaço virtual da "R.A.I.O" para a 
produção do cuidado em saúde, se comprometem a dar continuidade aos trabalhos, coordenando atividades de manutenção deste site, bem como sensibilizando a gestão da Unidade de Oncologia/UFTM para o fortalecimento desta ação.

Entende-se que cabe um esforço coletivo para a divulgação do site e o envolvimento de outros atores de interesse no tema para a construção colaborativa de um espaço virtual em formato de site e expressões de emoções e sentimentos, produção de cuidado integrado e interação social, e a criação de rede de apoio/suporte à pessoa com diagnóstico de câncer, uma vez que a internet vem se configurando como um espaço para a otimização do autocuidado em saúde e empoderamento da população.

\section{Conclusão}

Este estudo demonstrou que a criação de um site colaborativo e temático sobre o câncer pôde contribuir para facilitar o acesso à informação qualificada sobre o tema, bem como para a revisão da relação terapêutica entre profissionais do âmbito da saúde e usuários da unidade de oncologia pesquisada. Ademais, deu ênfase sobre a importância dos temas religiosidade e espiritualidade no processo de adoecer por câncer, visto que são recursos essenciais e devem ser levados em consideração pelos profissionais de saúde no processo de proporcionar a humanização do cuidado aos pacientes com câncer.

Com base nos resultados desse estudo, percebeu-se que promover espaços de socialização e produção de vida, ainda que no ambiente virtual, tente a contribuir para agregar benefícios para a melhoria da sistematização da atenção em saúde, além de promover o autocuidado e a autogestão em saúde no que se refere à área da oncologia.

Neste contexto, é de suma importância que todos os envolvidos no cuidado à pessoa com câncer compreendam e se atentem ao fato de que o adoecer por esta doença é um fenômeno complexo, multidimensional, que perpassa pelos aspectos biológicos, psicológicos e sociais. Faz-se necessário desenvolver ações terapêuticas envolvendo diversas categorias profissionais de caráter interdisciplinar que levem em consideração as subjetividades de cada indivíduo, seus anseios e desejos, no intuito de contribuir para a integralidade da saúde desses indivíduos e de seus familiares.

Enfatiza-se que a utilização da internet para o cuidado e acesso à informação em saúde tem sido mais frequente na sociedade contemporânea, impulsionando a atenção à saúde para este fenômeno, abordado com vistas a incorporá-lo no cuidado qualificado em saúde. 


\section{Agradecimentos}

A Coordenação de Aperfeiçoamento de

Pessoal de Nível Superior (CAPES) pelo incentivo em pesquisa e aprimoramento em nível superior.

Apoio financeiro: Gerência de Ensino e Pesquisa/HC-UFTM - Filial EBSERH Edital no 02/2017.

\section{Referências}

1. Silva A, Ferreira M. Gestão do conhecimento e capital social: as redes e sua importância para as empresas. Informação \& Informação., Londrina, v. 12, n. esp., p.1-32, 2007.

2. Ferreira GC. Redes sociais de informação: uma história e um estudo de caso. Perspectivas em Ciência da Informação. Belo Horizonte, v.16, n. 3, p. 208-231, 2011.

3. Castells MA. sociedade em rede. Rio de Janeiro: Jorge Zahar, 1999.

4. Andrade GRB, Vaitsman J. Apoio social e redes: conectando solidariedade e saúde. Ciênc. saúde coletiva., Rio de Janeiro, v. 7, n. 4, p. 925-934, 2002.

5. Domingues DF, Dessen MA. Reorganização familiar e rede social de apoio pós-homicídio juvenil. Psicologia: Teoria e Pesquisa., Brasília, v. 29, n. 2, p. 141-148, abrjun. 2013.

6. Abers R, Bulow MV. Movimentos sociais na teoria e na prática: como estudar o ativismo através da fronteira entre Estado e sociedade?

Sociologias, Porto Alegre, v.13, n. 28, p. 52-84, 2011.

7. Stolagli VP, Evangelista MRB, Camargo OP. Implicações sociais enfrentadas pelas famílias que possuem pacientes com sarcoma ósseo. Acto Ortop. Bras., São Paulo, v. 16, n. 4, p. 242-246, 2008.

8. Freitas MC, Mendes MMR. Condição crônica: análise do conceito no contexto da saúde do adulto. Rev Latino-am Enfermagem., Ribeirão Preto, v. 15, n. 4, p.1-8, jul/ago.2007.

9. Nicolussi AC, Sawada NO. Fatores que influenciam a qualidade de vida de pacientes com Câncer de cólon e reto. Acta Paul Enferm., São Paulo, v. 23, n. 2, p. 125-130, 2010.

10. Peruzzo CMK. Da observação participante à pesquisa-ação em comunicação: pressupostos epistemológicos e metodológicos. INTERCOM - sociedade Brasileira de Estudos Interdisciplinares da comunicação [s.n], 2003.

11. Thiollent M. Metodologia da pesquisaação. São Paulo: Cortez, 1947.

12. Cohen S. Social relationships and health. American Psychologist. November, 2004.

13. Orem DE. Nursing: concepts of practice. 6aㅡ ed, Mosby, 2001.

14. Wen CL. Telemedicina e telessaúde: inovação e sustentabilidade. $50^{\circ}$ congresso científico do HUPE, 2012. Disponível em:

http://www.telessaude.uerj.br/goldbook /artigos_busca/> acessado em: 2 de março de 2019.

15. Lévy P. As tecnologias da inteligência. São Paulo: Editora 34, 1999.

16. Lopes AAF. Cuidado e empoderamento: a construção do sujeito responsável por sua saúde na experiência do diabetes. Saúde Soc., São Paulo, v. 24, n. 2, p. 486-500, 2015.

17. Gillioli G. O pequeno médico. 1 ed. São Paulo: Clio editora, 2007. 
18. Morais GB et al. A valia do vínculo na relação equipe multidisciplinarpaciente oncológico para a continuidade do cuidado: uma revisão integrativa. Revista Saúde e Ciência online., Campina Grande, v. 7, n. 2, 2018.

19. Yalom ID, Leszcz M. Psicoterapia de grupo: teoria e pratica. Porto Alegre: Artmed;2006.LINK1-disponível em:<http://www2.ebserh.gov.br/web/h c-uftm/oncologia-e-hematologia>. Acessado em: 2 de março de 2019.

20. Lima TO, Borges GC. Avaliação da qualidade de vida em pacientes com câncer assistidos pelo centro de tratamento de câncer de Dourados. Interbio. 2009.

21. Ministério da Saúde-MS. Consenso Nacional de Nutrição Oncológica Instituto Nacional de Câncer. Rio de Janeiro, RJ: MS, 2009.

22. Gianini MMS. Câncer e gênero: enfrentamento da doença. 2007. Dissertação de mestrado. Pontifícia Universidade Católica de São Paulo, São Paulo, 2007.

23. Giovanetti J. Psicologia existencial e espiritualidade. Psicologia e espiritualidade, ed paulus, São Paulo, 2005.

24. Pessini L. A Espiritualidade interpretada pelas ciências e pela saúde. O Mundo da Saúde, 2007.

25. World Health Organization. Constitution of the World Health Organization. Basic Documents [on line]. 45를 edition. Genebra: 2006. acessado em 13 out. 2017]. Disponível em: http://www.who.int/governance/eb/who _constitution_en.pdf.

26. Puchalski CM. The hole of spirituality in health care. BUMC Proceedings., Waco, v. 14, n. 4, p. 352-357, 2001.
27. Oliveira EA, Santos MA. Apoio psicológico na terminalidade: ensinamentos para a vida. Psicologia em Estudo, 2010. 


\section{Anexos}

Figura 1: Sessão "Álbum de Histórias".

$R$ Ain

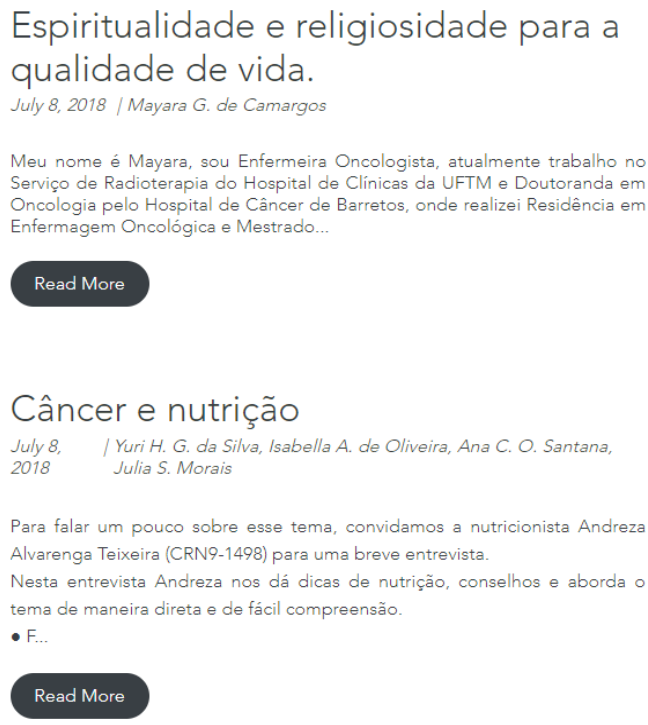

Our Recent Posts

Espiritualidade e religiosidade

para a qualidade de vida.

July 8, 2018

Câncer e nutrição

July 8, 2018

Trajetória de vida

June 4, 2018

Archive

July 2018

June 2018

April 2018

Tags

Fonte: www.raiooncologia.wixsite.com/raio

Figura 2: Sessão "Cuidando de mim".

$$
R \text { Ain }
$$

\section{Cuidando de mim}

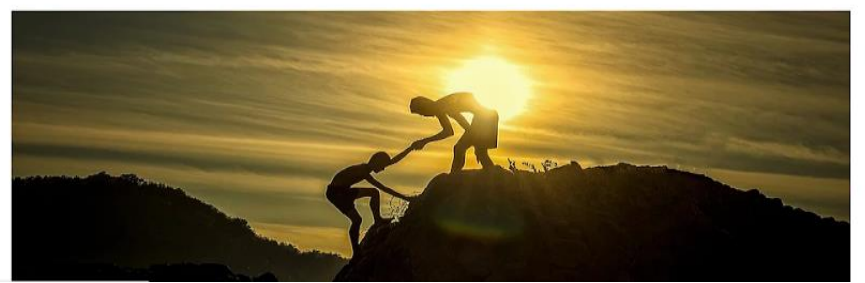

Fonte: www.raiooncologia.wixsite.com/raio 
Figura 3: Sessão "Buscando mais Informação"

$$
R \text { Alic }
$$

\section{Buscando mais informação}

Fonte: www.raiooncologia.wixsite.com/raio

Figura 4: Sessão "Depoimentos"

$$
\text { RAR }
$$

\section{Depoimentos}

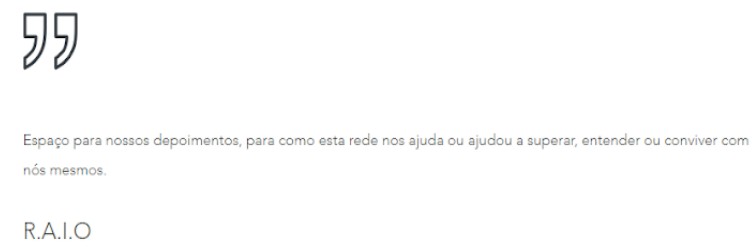

Fonte: www.raiooncologia.wixsite.com/raio 
Figura 5: Sessão "Contato"

$R A_{i}$

\section{Faça sua pergunta, entre em contato, nos escreva}

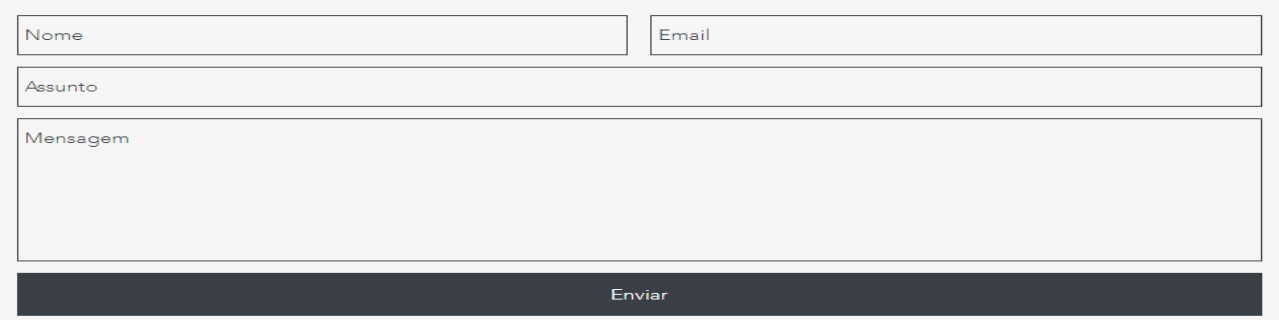

Fonte: www.raiooncologia.wixsite.com/raio 


\section{Como citar este artigo}

Silva YHGda, Frizzo HCF. R.A.I.O - espaço virtual para a construção colaborativa do cuidado em saúde no processo de adoecer por câncer. [online], volume 4, n. 1. Editor responsável: Luiz Roberto de Oliveira. Fortaleza, mês e ano, p. 85-101. Disponível em: http://periodicos.ufc.br/resdite/index. Acesso em "dia/mês/ano".

Data de recebimento do artigo: 10/04/2019

Data de aprovação do artigo: 25/06/2019 